\title{
Flexibility in component manufacturing systems: evaluation framework and case study
}

\author{
Dominic P. Hauser • Olivier L. de Weck
}

Received: January 2006 / Accepted: August 2006 / Published online: July 2007

(C) Springer Science+Business Media, LLC 2007

\begin{abstract}
Traditional component manufacturing systems have been optimized for either small scale craft production or for mass production of a small variety of high volume parts. Trends towards intermediate volumes and larger variety of parts have exposed the need for intelligently embedding flexibility in manufacturing systems and processes. The literature offers only few attempts to value component fabrication flexibility in a systematic way. In this article a 5-step framework for valuing flexibility and ranking of manufacturing processes under uncertainty is developed. A discrete time simulation is used to predict profit, remaining tool value and machine utilization as a function of three probabilistic demand and specification scenarios. A case study demonstrates the simulation and contrasts both a high volume (automotive) and a low volume (aerospace) market situation across six different processes ranging from punching to laser cutting. It is found that for intermediate, uncertain production volumes alternative manufacturing processes that embed flexibility carefully in one or more dimensions can outperform traditional processes that are either completely nonflexible (e.g., stamping) or completely flexible (e.g., laser cutting). It is also shown that flexibility in parts manufacturing is a complex topic because flexibility can be embedded in the parts themselves, in tooling or in the process parameters.
\end{abstract}

D. P. Hauser

Swiss Federal Institute of Technology, Zurich, Switzerland

Present Address:

D. P. Hauser

ThyssenKrupp Presta AG, Eschen 9492, Fuerstentum Liechtenstein

O. L. de Weck $(\varangle)$

Department of Aeronautics and Astronautics, Engineering

Systems Division (ESD), Massachusetts Institute of Technology,

Cambridge, MA, 02139, USA

e-mail: deweck@mit.edu
Keywords Manufacturing flexibility $\cdot$ Modular tooling . Process evaluation $\cdot$ Parts fabrication · Simulation

\section{Introduction}

Typically, component or parts fabrication is associated with a number of manufacturing processes such as stamping, milling or laser cutting. The traditional metrics for assessing competing processes are quality, cost and production rate. Forming technology and machining, e.g., can be compared based on process capability, fixed cost for equipment and tooling as well as per unit cost as a function of production volume. Increasingly, however, market driven uncertainties are starting to dominate the design and selection of fabrication processes. To cover a wide spectrum of characteristics between extreme markets, such as the automotive and the aerospace industry, special consideration must be given to flexibility. The aerospace industry on one side is characterized by low production volumes and large volatility in year-to-year demand. On the other side is the automotive market with high volumes and rather precise production forecasts. Consideration of those two extreme markets will help approach the problem of valuing flexibility from different sides.

Intermediate production volumes are increasingly important due to trends such as market fragmentation, mass customization (Pine, 1993) and product platforming (Simpson, Siddique, \& Jianxin, 2006). Two types of exogenous uncertainties are of particular interest: demand fluctuations and component specification changes. We will argue that these two aspects have exposed the need for embedding more flexibility in manufacturing systems and processes. The questions are then where and how much flexibility is needed, and how 


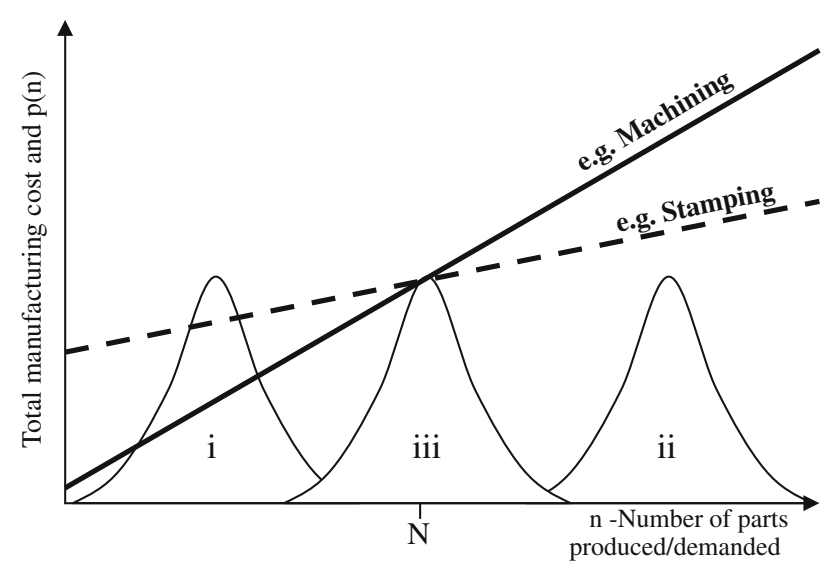

Fig. 1 Manufacturing cost curves and demand distribution

much benefit and cost can be expected under various production capacity and component specification scenarios.

\section{Capacity}

The capacity of a production facility can be measured as the number of units that can be produced per time unit. Sizing of capacity is primarily driven by the expected demand for particular types of components. Craftsmanship-type processes are flexible, but only competitive for small volumes. Highly automated processes have high capacity, but require large investments in tooling and machinery and are only economical for large volumes.

In Fig. 1 hypothetical cost curves depending on the number of produced parts of two manufacturing process technologies (e.g., stamping and machining) are compared. Stamping has higher fixed costs for tooling than machining, but therefore, has lower variable production costs, especially at a higher output rate. So, there exists an intersection point at production volume $N$. If the expected distribution of the demand $p(n)$ is like in case i (see Fig. 1), it is clear that a firm will use the 'machining' process. On the other hand, if the demand is distributed as shown in case ii, there is no question that 'stamping' will be more profitable. The crucial question now is, which process technology should be chosen if the demand is distributed around $\mathrm{N}$ (demand distribution iii $=$ intermediate volume situation). One possible solution to this problem is to search for a new process whose total costs at the production quantity $\mathrm{N}$ are lower than those of the other two processes.

\section{Component specifications}

The primary specifications of structural components consist of geometrical dimensions, material selections, surface finish and tolerances among others. A bicycle manufacturer, for example, will carefully specify the frames of the bicy-

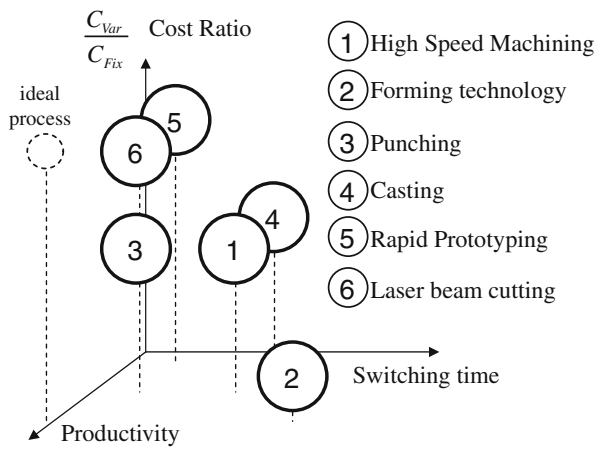

Fig. 2 Manufacturing processes and the tradeoff between productivity, switching time and cost structure

cles using these attributes. However, different parts may be used to fulfill specific functions in their 'racing', 'mountain' and 'recreational' bike models. Such variety in models requires frequent part and specification changes, in addition to lower and more uncertain production volumes of standardized parts. Some manufacturing processes are inherently more flexible than others in terms of accommodating such specification changes.

In Fig. 2 high speed machining, forming technology, punching, casting, prototyping, and laser beam cutting are compared qualitatively to each other according to their process switching time, their productivity and their ratio of variable to fixed costs. We know from experience that the more to the left a process is located in Fig. 2, the easier switching from manufacturing one part to another can be (important for a large product mix). The higher a process is placed in this chart the easier new parts can be added to the current product range (important for a fast changing product mix). Processes placed lower on that axis require more fixed investments, specifically for tooling.

The third dimension, productivity, captures how many units per time can be manufactured with a given process technology and machine. The further towards the front a process is located, the higher its output rate. An ideal process would be located in the upper left front corner of this chart. Which of these processes should be chosen in a given situation? Can there be other processes that strike a careful compromise between efficiency and flexibility and might be superior to the ones shown? Finding answers to these questions is the purpose of this paper.

In the next section we first give a brief overview of relevant literature in the area of flexible component fabrication. This will clarify different types of flexibility, but does not help us value and decide whether flexibility is worthwhile implementing in a particular situation. Section 'Flexibility Evaluation Framework' introduces a 5-step flexibility evaluation framework for parts manufacturing. The approach is demonstrated in Section 'Case study' using a simple case 
study with three uncertain production scenarios. We summarize the results in Section 'Conclusions' followed by recommendations for future work in Section 'Future Research'.

\section{Literature review}

In order to analyze the influence of the characteristics mentioned above, definitons for flexibility should first be given. Fricke et al. (2000) and Schulz and Fricke (1999) define flexibility as a system's ability to change easily. More specifically, flexibility is defined by Upton (1994) as 'the ability to change or react with little penalty in time, effort, cost, or performance'.

Different types of flexibility can be distinguished. According to Gupta and Somers (1992), one of the 'most thorough classification systems' for flexibility was proposed by Sethi and Sethi (1990). As they put it, flexibility has been widely recognized as a multi-dimensional concept within the manufacturing function. They distinguish among eleven types of flexibility whose definitions will be given here in brief:

- Machine flexibility (of a machine) refers to the variety of operations that a machine can perform without requiring a prohibitive effort in switching from one operation to another.

- Flexibility of a material handling system is its ability to move different part types efficiently for proper positioning and processing through the manufacturing facility it serves.

- Operation flexibility of a part refers to its ability to be produced in different ways.

- Process flexibility of a manufacturing system relates to the set of part types that the system can produce without major setups.

- Routing flexibility of a manufacturing system is its ability to produce a part by alternate routes through the system.

- Product flexibility is the ease with which new parts can be added or substituted for existing parts.

- Volume flexibility of a manufacturing system is its ability to be operated profitably at different overall output levels.

- Expansion flexibility of a manufacturing system is the ease with which its capacity and capability can be increased when needed.

- Program flexibility is the ability of the system to run virtually untended for a long period.

- Production flexibility is the universe of part types that the manufacturing system can produce without adding major capital equipment.

- Market flexibility, finally, is defined as the ease with which the manufacturing system can adapt to a changing market environment.
Flexibility in manufacturing has also been researched extensively by an NSF Engineering Research Center at the University of Michigan. (Mehrabi, Ulsoy, \& Koren , 2000) for example developed a control theoretic approach to demonstrating the value of reconfigurable manufacturing systems.

An approach dealing with volume flexibility was proposed by Mills (1984). He developed a quadratic cost function

$c_{t}(n)=\alpha+\beta n+\frac{n^{2}}{2 \delta}$

where $c_{t}$ is the total cost, $n$ is the production volume, and $\alpha$, $\beta$, and $\delta$ are positive constants. The average costs per part are therefore

$c_{a}(n)=\frac{1}{n} c_{t}(n)=\frac{\alpha}{n}+\beta+\frac{n}{2 \delta}$

and the marginal costs, as the derivative of the total costs with respect to the number of units $n$, are positive and increasing

$c_{m}(n)=\frac{\partial c_{t}(n)}{\partial n}=\beta+\frac{n}{\delta}$

As can be seen, e.g., in (Schips, 2000), average costs decrease with increasing production volume $n$ as long as they are higher than marginal costs. Therefore, the crossover point of average and marginal costs is coexistent with the optimal output volume. At that specific production quantity the average costs are lowest. This circumstance can also be shown by analyzing Mills' cost function. The average costs' low point is at

$\frac{\partial c_{a}(n)}{\partial n} \stackrel{!}{=} 0 \Longrightarrow n=\sqrt{2 \alpha \delta}$

and for the intersection of the average and the marginal cost curves the same volume is found

$c_{a}(n) \stackrel{!}{=} c_{m}(n) \Longrightarrow n=\sqrt{2 \alpha \delta}$

This formula can now be used to determine the optimal output (optimal in the economic sense) of an existing or planned production facility or process. Knowing the machine's average cost curve, Mills' quadratic cost function can be approximated to the 'real' cost curve by estimating values for the parameters $\alpha$ and $\delta$. Then, the optimal production state can easily be calculated. However, even if an 'optimal' production system were established according to Eq. (5), it is inevitable that the actually required production quantity $n$ will change over time.

\section{Flexibility evaluation framework}

So, how can flexibility be evaluated in general? An important question for a manufacturing firm is how the value of 


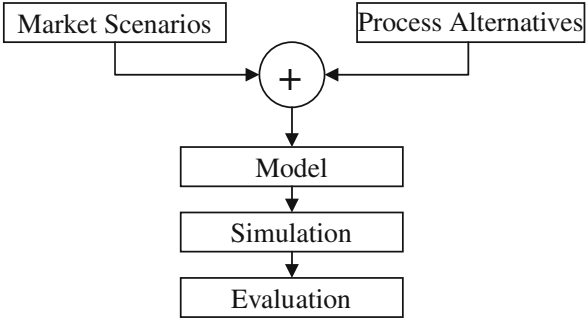

Fig. 3 Steps in the approach to value flexibility

flexibility can be evaluated in a strategic production planning process. Here, an approach which contains five major steps, see Fig. 3, is suggested.

The steps are as follows:

1. Building a set of market scenarios of possible future market evolution. Such scenarios must provide information from outside the firm. A scenario contains the number of different products, their expected sales volumes, geometry data, and expected sales prices.

2. Defining process alternatives for new possible production processes as well as for at least one existing baseline process. These process alternatives should be characterized by different types and degrees of flexibility. A process alternative has to cover information about the output rate of the system, the machine cost and its lifetime, the costs for tools and their lifetimes, the switching costs which originate from setting-up the system for another product to be produced on the system, the various variable costs such as labor, energy and supplies, the raw material utilization, and the scrap rate.

3. Creating a model in which the behavior of the firm can be simulated depending on the diverse combination of market scenarios and process alternatives. In that model general statements like costs for inventory holding and overdue parts, but also costs for overhead, number of workdays per year, shifts per day, and effective work hours per shift, and material costs per unit are captured. In this step a discrete time simulation should be defined to generate random orders (within the boundaries defined in the market scenario) for the parts.

4. Running the discrete time simulation for every combination of market scenario and process alternative.

5. Finally, the evaluation of the simulation results leads to a ranking of the most adequate manufacturing process for the firm. This step should also include a sensitivity analysis to understand the robustness of the ranking.

\section{Case study}

To illustrate the application of this procedure, a case studyusing a family of simple metallic components (levers) as
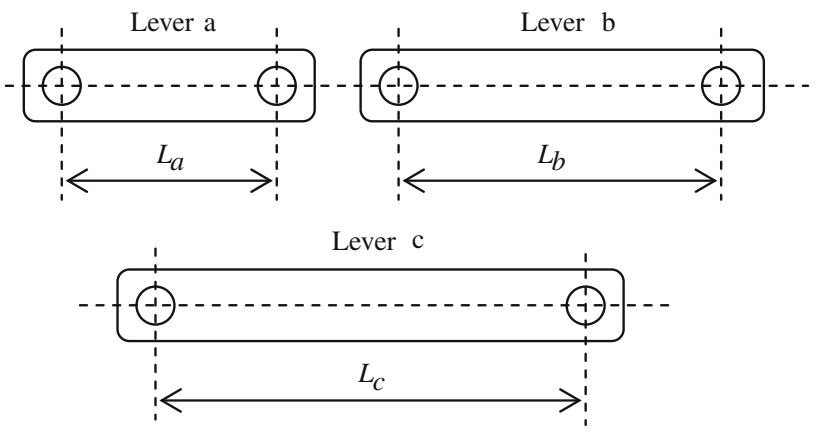

Fig. 4 Three metal levers: a, b, c

hypothetical parts-is presented here. We will explain the fundamental flexibility problem with the aid of two virtual companies, $A$ and $B$, that are producing and selling these levers. We note that the analysis methodology developed for this example is generalizable to other situations.

\section{Problem formulation}

Company $A$, a small manufacturing firm, fabricates and sells simple levers $a, b$, and $c$ for the automotive industry. The levers have identical width, two holes each, and different lengths as shown in Fig. 4.

The company currently offers this family of three levers in three different lengths: $L_{a}=0.5<L_{b}=0.75<L_{c}=1.0$. The lengths have been determined by the firm's customers, but might be subject to change in the future based on shifting customer preferences. Currently the company is manufacturing and selling the following number of units per year: $S_{a}=200,000, S_{b}=300,000$, and $S_{c}=100,000$. These sales numbers strongly fluctuate from year-to-year. Lever $a$ is sold for $\$ 1.00$ per piece, lever $b$ for $\$ 1.50$ per piece and the price for lever $c$ is $\$ 2.00$ per piece. No volume discounts are granted.

The current manufacturing process is stamping, which requires a special, dedicated tool (die) for each part $a, b$, and $c$. Each tool has an estimated lifetime of 1,000,000 stampings and all of them have been newly fabricated at the beginning of the current year. The capital investment cost for the stamping machine is $\$ 5,000,000$; the lifetime of the machine is 10 years.

Company $B$, also a small manufacturing firm, but serving the aerospace industry, produces exactly the same components than firm $A$. Since the aerospace industry has different requirements than the automotive industry, the two firms are not competing with each other. Due to lower production volumes, and supposed higher quality levels, firm $B$ can sell its lever $a$ for $\$ 15.00$ per piece, lever $b$ for $\$ 22.50$ each, and lever $c$ for $\$ 30.00$ per part. On the other hand, the production volumes are relatively low with $S_{a}=2,100, S_{b}=3,750$, 


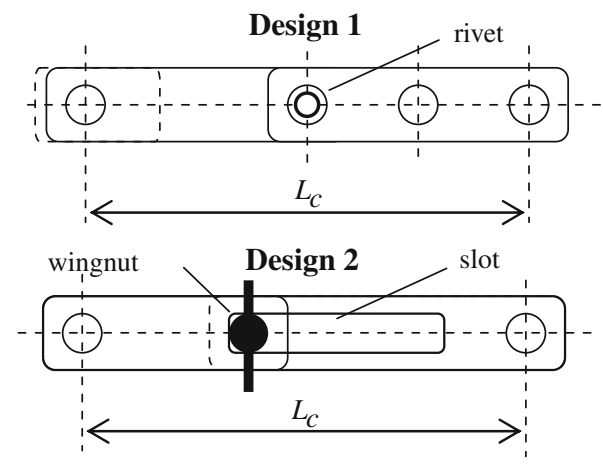

Fig. 5 Two competing designs for imparting lever (product) flexibility

and $S_{c}=1,150$ units. All the other settings are assumed to be the same as for firm $A$.

The marketing departments of the two firms generate predictions about next year's sales (market scenarios): There is a $50 \%$ chance that next year's parts will be the same as this year's. There is a $25 \%$ chance that a new part $d$ with $L_{d}=0.875$ will be added to the parts family $a, b$, and $c$. There is a $25 \%$ chance that an entirely new lever product line $\alpha, \beta$, and $\gamma$ with $L_{\alpha}=0.4, L_{\beta}=0.8$, and $L_{\gamma}=1.2$ will have to be developed to replace the current lever family. The expected sales volumes for next year are up by $5 \%$, $25 \%$ and $15 \%$ for $a, b$ and $c$, respectively but are subject to uncertainty. Sales volumes for $\alpha, \beta$, and $\gamma$ would fluctuate uniformly between 50,000 and 500,000 per part in the automotive industry and between 0 units and 5,000 units per part for firm $B$. This statement captures both demand and product specification uncertainty.

How can the design or manufacturing of these parts be improved to make the overall system more flexible and competitive in the face of future specification (geometry) and demand uncertainties? This requires consideration of both engineering as well as economic aspects of the problem.

The engineering teams propose two solutions. Both of them offer the possibility to adapt the length of the lever individually, see Fig. 5.

The first design divides the lever into two pieces and adds a set of holes at fixed locations. The two parts are partially overlapped and riveted together to achieve the desired length. The second design also uses two parts, but introduces a slot in the second part and connects the parts with a wing nut. The first design allows some discrete flexibility for the manufacturer to choose the length but adds complexity (two parts instead of one) and some costs (addition of the riveting process). The second design gives flexibility to the customer, but adds weight (wing nut) and complexity to the part. Here, flexibility is embedded in the product directly. It is unclear whether the customer will be willing to accept the new design and what the exact impact on the manufacturing costs (fixed and variable) and profits will be. So, flexible solutions in manufacturing rather than in design are investigated.

Which process technologies can be used for manufacturing the aforementioned parts? What are the earnings, the risk, and opportunity (e.g., free capacity on machines) depending on the chosen manufacturing process? What are the differences between technologies for firm $A$ and firm $B$ ?

\section{Market scenarios}

The first step in the framework, Fig. 3, is to generate realistic market scenarios. Three possible situations may occur next year, see Fig. 6.

These scenarios are named Scenario A for the most likely case in which the products are the same as this year, Scenario $B$ for the situation in which a fourth lever $d$ is added to the three existing levers, and Scenario $C$ for the event when the entire product line is replaced by a new one (greek letters). These three scenarios indicate different levels of complexity and uncertainty for the firm. They are ordered in increasing order of technical and managerial challenge.

Process alternatives

Six possible process alternatives (second step in Fig. 3) are described here. They are named Process I-VI. The processes distinguish themselves basically by the following parameters:

1. The output rate $r_{\text {Output }}$ stands for the capacity per unit of time and is measured in parts per minute on a single machine

2. The machine costs $C_{M}$ in $\$$ are the initial capital costs for the acquisition of the machine

3. The machine's lifetime $T_{L M}$ in years is needed for the depreciation of the machine

4. The tool's lifetime $T_{L T}$ measured in the quantity of parts that can be produced with one tool before it must be replaced is also needed for the depreciation rate

5. The switching time $T_{S}$ in hours is needed to set up the machine for another product

6. The variable costs $C_{v_{H}}$ include wages, costs for energy and other supplies

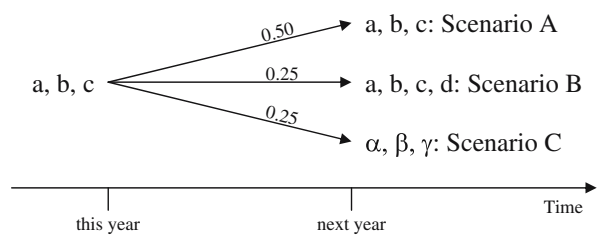

Fig. 6 Three probabilistic market scenarios for the following time period 


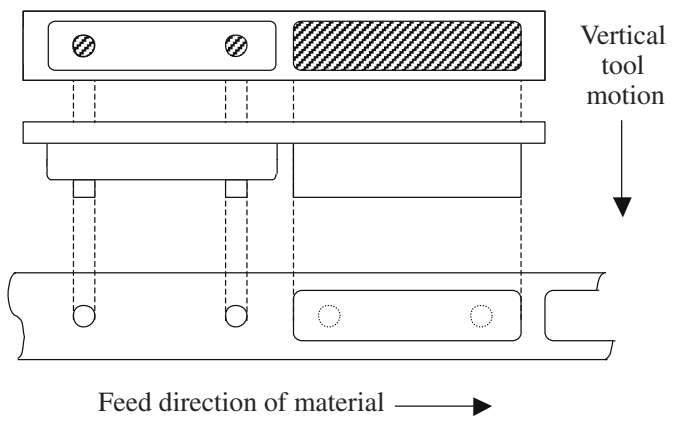

Fig. 7 Tool for Process $I$ and the metal feedstock band

7. The raw material utilization $r_{M a t}$ indicates whether the process requires material that cannot be used afterwards (waste material), e.g., for gripping areas

8. The scrap rate $r_{S c r a p}$ defines how many of the produced parts must be thrown away because they do not meet quality requirements

9. The costs for common tools $C_{T c}$, measured in $\$$, can be depreciated over all products when common tools are used for the production of all parts

10. The costs for the dedicated tools $C_{T d}$ for each lever, also given in $\$$, can only be amortized by producing a specific part

The details of these process alternatives are discussed in the next paragraphs.

Process $I$ describes the current baseline manufacturing process in which a dedicated tool is used for each part. In a two-step progressive process, first the holes are punched, and then in the second step the contour of the lever is cut. See Fig. 7 for a schematic of the tool.

The raw material is a metal band. The tool can be exchanged as one whole block and so the switching time is rather low. The scrap rate is very low due to the dedicated tool, so it is set to zero. The tool costs are assumed as $\$ 150,000$ for the smallest part and then increase with the length of the lever. The output rate is set to 50 parts per minute.

The second process alternative, Process II, takes a first step toward 'modular tooling'. That means that parts of the tool are reused by all, or at least several, product variants while other parts of the tool are dedicated to only one variant.

As shown in Fig. 8 the end parts and the middle part of the tool are separated. While the end sections of the tool can be used and adjusted with every lever, the middle part is changed to match the needed length. For this more complicated operation, compared to Process I, the switching time for this process is doubled. The scrap rate is set to $1 \%$ due to the fact that the probability of failure in the process is increasing with the additional degree of freedom, which is embedded in the tool. The manufacturing process itself is the same as in Process $I$.

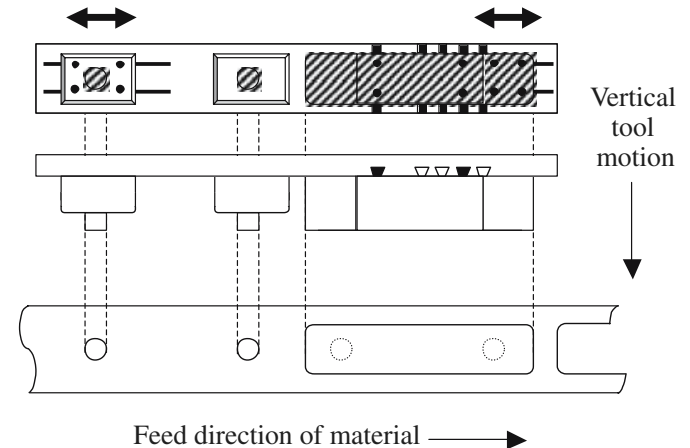

Fig. 8 Modular tool for Process II and the metal feedstock band

In the third process alternative (Process III), the levers are produced using a laser beam cutting machine. The raw material is sheet metal, therefore the material utilization is reduced to $90 \%$ because of the areas needed to hold the sheet. The capacity here is very low, but on the other hand costs for tooling are not incurred. The variable costs are high because the efficiency factor of today's lasers is still low, so energy costs are very high.

For the punching process, Process $I V$, raw material is also sheet metal. But, more area is needed for gripping due to the fact that the sheet has to be moved relative to the punching apparatus, while in process III the head of the laser moves relative to the sheet. As improved process control is assumed relative to laser beam cutting, the scrap rate is lower than in the laser cutting process above.

Milling in Process $V$ is the slowest of all process alternatives. However, the variable costs are low (needs less energy than laser beam and only few auxiliary materials).

The last process alternative, Process VI, introduces a new paradigm. The basic process is, as in the first and second process, stamping. But now, not the tools are changed to adapt the process to the parts, but the process parameters for feeding of raw material are dependent on the product.

As shown in Fig. 9 the tool makes the front part of one lever in the same production cycle as the rear portion of the preceding component. To adjust the process to the product, a dead stop is needed to control the feeding of the band material. Thus, flexibility is embedded in the process parameters, rather than in the tooling or product itself. It is necessary that the raw material has already the correct width, because only the end parts of the levers will be processed. Process VI is a special purpose process, whereby flexibility is embedded in one specific dimension (length) only.

Model and simulation

The model (see Fig. 3) consist of a simulation, which simulates the demand for each part per period, and an algorithm, which computes the production costs and the firm's earnings 


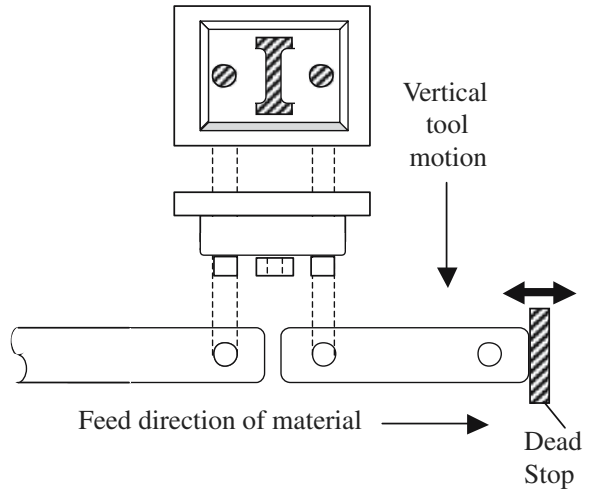

Fig. 9 The tool for Process VI ("infeed") and the metal feedstock band

for every combination of uncertain market scenario $(A, B$, $C)$ and process alternative $(I-V I)$. In this model, a period is assumed as one day and the simulation runs for 1 year, which corresponds to 250 working days. It is assumed that the manufacturing is set up as single thread on a single machine.

Except in the case of market scenario $C$, in which the demand will fluctuate uniformly between a lower and an upper bound, the daily demand is assumed to be normally distributed with a mean value which is equal to one day's part of the annual demand. The standard deviation for this normal distribution is set to 200 parts per day for the automotive industry and 10 parts per day for the aerospace industry.

Figure 10 shows the main flow diagram of the simulation.

The randomized demand $\left(D_{t}\right)$ is generated by the simulation tool. For determining the target production lot size for the current period $\left(P_{T, t}\right)$, overdue parts from the last period ( $\left.n_{\text {Overdue }, t-1}\right)$ are added and parts in stock from the last period $\left(n_{\text {Stock,t-1 }}\right)$ are subtracted. The target production batch is either zero (when the actual demand is smaller than the available amount of pieces in stock) or the actual demand is corrected by the number of stock and overdue parts

$P_{T, t}=\max \left(0, D_{t}-n_{\text {Stock }, t-1}+n_{\text {Overdue }, t-1}\right)$

The available capacity bounds the actual lot size. It can be calculated as the available capacity of the current period $\left(C A P_{t}\right)$ minus the already booked capacity of the current period. If the feasible production volume $\left(P_{A, t}\right)$ plus stock from the last period is less than demand, parts become overdue. If the feasible production is higher than demand plus the amount of overdue parts from the last period, parts go to stock. Hence, the target production must be compared with the remaining capacity of the production period.

$$
P_{A, t}=\min \left(P_{T, t}, C A P_{t}-\sum_{\text {otherparts }} P_{A, t}\right)
$$

In Eq. 7 the remaining capacity is calculated as the difference between the total capacity per period and the capacity volume already spent for producing other parts in that period.

In one period, the firm can sell either as many parts as are demanded in the current period plus those which are backlogged from the last period, or as many as it produced in the current period plus those which are available in stock from the last period.

$S_{t}=\min \left(D_{t}+n_{\text {Overdue }, t-1}, P_{A, t}+n_{\text {Stock }, t-1}\right)$

In the model, the firm has to pay a penalty for overdue parts, by reducing the price charged by a percentage which increases with the number of periods overdue. Also, for pieces in stock the firm reduces its profit due to inventory holding costs. To minimize inventory keeping, the machine will be set up for every product once per period. So, the remaining question for scheduling is in which order the parts should be produced, or, if necessary, which parts should be made overdue. To answer this question the contribution margins of the products are compared. The product with the highest margin will be produced first, the product with the lowest margin is produced last.

Combining the three market scenarios $(A, B, C)$ with the six process alternatives $(I-V I), 18$ different cases can now be analyzed. The results of the calculations themselves are subject to uncertainty, because of the simulation procedure with randomly generated demand. To obtain a statistically significant expected value for the results, every case is simulated 100 times using the Monte Carlo technique.

Three metrics, Total earnings, Tool value, and Machine utilization are defined to be most important and are analyzed for every simulation run. Total earnings captures the firm's profit (or loss) and is calculated as the non-discounted difference between revenues and costs.

$E_{T o t}=\sum R_{t}-\sum C_{T o t, t}$

The metric Tool value describes the residual value in the tools at the end of the simulation time period. It is derived from the tool costs and the tool depreciation.

$V_{T}=\sum_{\text {tools }} C_{T}\left(T_{L T}-\sum_{\text {periods }} D_{T, t}\right)$

If, due to product changes, a tool has to be replaced before the end of its life, the capital investment in the tool is lost. Therefore, a low Tool value, $V_{T}$, at the end of the simulation is desirable as this indicates good amortization and lower risk.

The Machine utilization indicates the fraction of time during which the machine is used to produce the parts family. 


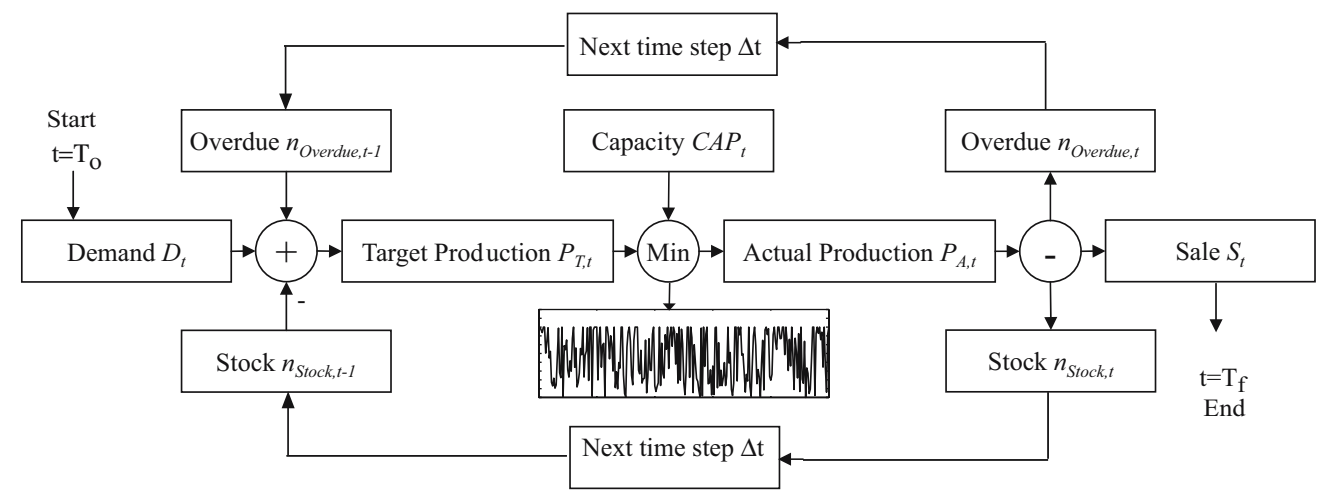

Fig. 10 Main flow diagram of the manufacturing simulation

\begin{tabular}{|c|c|c|c|c|c|c|c|c|}
\hline & & & \multicolumn{6}{|c|}{ Process Scenarios } \\
\hline & & & $\mathrm{I}$ & II & III & IV & $\mathrm{V}$ & VI \\
\hline & \multirow{7}{*}{ 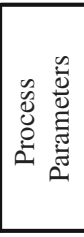 } & Output [pcs / $\mathrm{min}$ ] & 50 & 50 & 4 & 10 & 1 & 30 \\
\hline & & Switching time $[\mathrm{h}]$ & 0.25 & 0.5 & 0 & 0 & 0 & 0.05 \\
\hline & & Variable production costs [\$] & 20 & 20 & 50 & 30 & 30 & 20 \\
\hline & & Raw material utilization & $100 \%$ & $100 \%$ & $90 \%$ & $85 \%$ & $100 \%$ & $100 \%$ \\
\hline & & Scrap rate & $0 \%$ & $1 \%$ & $5 \%$ & $2 \%$ & $2 \%$ & $1 \%$ \\
\hline & & Costs for common tool [\$] & & 100,000 & 10,000 & 20,000 & 25,000 & 150,000 \\
\hline & & Costs for dedicated tool [\$] & 150,000 & 75,000 & & & & \\
\hline \multirow{3}{*}{$\begin{array}{c}\text { Market } \\
\text { Scenarios: } \\
\text { Automotive } \\
\text { Firm A } \\
\text { High Volume } \\
\end{array}$} & A & $\begin{array}{l}\text { a: } 210,000 \mathrm{pcs}, \$ 1 ; \mathrm{b}: 375,000 \mathrm{pcs}, \\
\$ 1.50 ; \mathrm{c}: 115,000 \mathrm{pcs}, \$ 2\end{array}$ & (2) 151,656 & 56,292 & $-382,946$ & $-103,570$ & $-924,767$ & (1) 176,099 \\
\hline & B & $\begin{array}{l}\text { a: } 210,000 \text { pcs, } \$ 1 ; \text { b: } 345,000 \text { pcs, } \$ 1.50 \text {; } \\
\text { c: } 108,000 \text { pcs, } \$ 2 ; \text { d: } 87,000 \text { pcs, } \$ 1.75\end{array}$ & (2) 154,006 & 35,243 & $-351,511$ & $-103,671$ & $-979,027$ & (1) 195,362 \\
\hline & $\mathrm{C}$ & $\begin{array}{l}\text { all parts } 50,000-500,000 \mathrm{pcs}, \quad \alpha: \$ 0.90, \\
\beta: \$ 1.60, \gamma: \$ 2.40\end{array}$ & (2) 258,007 & 156,484 & $-367,908$ & $-60,228$ & $-867,368$ & (1) 299,040 \\
\hline \multirow{3}{*}{$\begin{array}{c}\text { Market } \\
\text { Scenarios: } \\
\text { Aerospace } \\
\text { Firm B } \\
\text { Low Volume }\end{array}$} & A & $\begin{array}{l}\text { a: } 2,100 \mathrm{pcs}, \$ 15 ; \mathrm{b}: 3,750 \mathrm{pcs}, \$ 22.50 ; \\
\text { c: } 1,150 \mathrm{pcs}, \$ 30\end{array}$ & 113,597 & 61,688 & 154,698 & (1) 162,817 & 119,318 & (2) 154,635 \\
\hline & B & $\begin{array}{l}\text { a: } 2,100 \text { pcs, } \$ 15 ; \text { b: } 3,450 \text { pcs, } \$ 22.50 \\
\text { c: } 1,080 \text { pcs, } \$ 30 ; \text { d: } 870 \text { pcs, } \$ 26.25\end{array}$ & 130,676 & 65,262 & 183,673 & (1) 93,019 & 142,985 & (2) 182,556 \\
\hline & $\mathrm{C}$ & $\begin{array}{l}\text { all parts } 0-5,000 \mathrm{pcs}, \quad \alpha: \$ 12, \quad \beta: \$ 24 \\
\gamma: \$ 36\end{array}$ & 107,720 & 44,662 & 160,757 & (1) 168,401 & 127,549 & (2) 57,864 \\
\hline
\end{tabular}

The following process parameters are assumed to be the same for all process scenarios: Machine Costs [\$]: 5,000,000; Machine lifetime [a]: 10; Tools lifetime [pcs]: 1,000,000
(1) Best process

(2) Second best process

Fig. 11 Overview of all simulation results (Expected Earnings in \$)

A high Machine utilization, therefore, stands for high opportunity costs, because no other products may be produced on that machine.

The three metrics will generally be in tension with each other, see also Fig.2

\section{Evaluation}

Once the problem has been set up and simulation performed for all 18 combinations of future market scenarios and process choices, the results need to be carefully evaluated. This is the last step in the 5-step method (Fig. 3). As mentioned at the beginning of this section, market scenario $A$ is the most likely case. Thus, its importance is greater than those of the other two market scenarios. So, only Scenario $A$ will be discussed in detail here. However, a complete overview of all simulation results is shown in Fig. 11. Additional details and discussion for the other market scenarios can be found in Hauser (2004).

\section{High volume situation (Automotive)}

In Subsection 'Problem formulation' an annual demand of 700,000 units was predicted. The results of the simulation show an average demand of 700,259 parts with a standard deviation of 5,308 pieces per year.

For every process the three evaluation metrics are displayed. Table 1 and Fig. 12 show the results for all of the process alternatives in market scenario $A$ in the case of firm $A$. For market scenario $A$, process $V I$ is the process with the highest earnings. In addition it features relatively low sunk capital in the tools at the end of the year. Regarding the earn- 
Table 1 Results of the simulation for Scenario $A$ in the automotive industry

\begin{tabular}{llll}
\hline Process & Earnings & Tool value & $\begin{array}{l}\text { Machine } \\
\text { utilization }\end{array}$ \\
\hline I & 151,656 & 349,692 & $13 \%$ \\
II & 56,292 & 313,571 & $13 \%$ \\
III & $-328,946$ & 4,146 & $83 \%$ \\
IV & $-103,570$ & 8,292 & $67 \%$ \\
V & $-924,767$ & 19,996 & $100 \%$ \\
VI & 176,099 & 62,189 & $22 \%$ \\
\hline
\end{tabular}

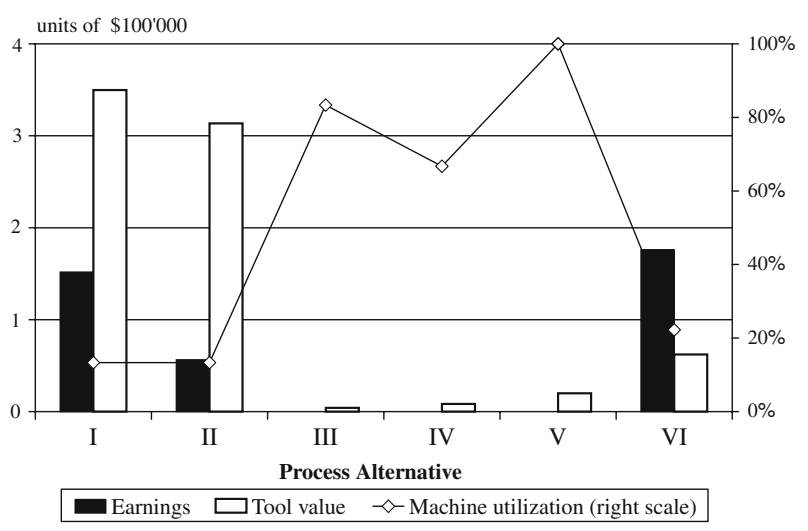

Fig. 12 Results of the simulation for scenario $A$ in the high volume situation (automotive industry)

ings, $V I$ is followed closely by process $I$, and, already with some distance, process II. The other three processes will not result in positive net earnings (see Table 1).

Reasons for the existence of this clustering into profitable and non-profitable processes in the high volume case are mainly twofold. First, laser beam cutting and milling (processes III and $V$ ) are, due to their low capacity, charged with significant overdue costs. The low capacity of those two processes can be seen clearly in Fig. 12 if one follows the line graph which indicates the machine utilization. Second, for punching (process $I V$ ), variable manufacturing costs per part are at such a high level that they cannot lead to positive profit. The higher manufacturing costs of these three technologies can be explained with the higher variable costs in combination with the lower output rate, i.e., higher costs per hour must be distributed among fewer parts produced per hour.

The cause for process $I I$ 's gap to $I$ and $V I$ is mainly due to the doubled switching time compared to scenario $I$. So, process $I I$ with modular tooling is not competitive in the high volume situation of market scenario $A$ due to switching costs.

Not only is process $V I$ a very good process technology in all market scenarios, it is also the only process scenario which has a 'earnings-to-tool value' ratio that is greater than one. This ratio can also be interpreted as an earnings-to- risk ratio. Process VI, having embedded flexibility, has both higher expected earnings and lower sunk costs in tooling than the more traditional non-flexible stamping process $I$.

A recommendation to firm $A$ 's management should be made to replace the current production technology by process VI. Since the new technology is also a stamping process, it should be possible to reuse the press. Some adaptations, like better feedstock guidance, better feed control, and the dead-stop (see Fig. 9), may be necessary.

\section{Low volume situation (Aerospace)}

Not surprisingly, the results look quite different for firm $B$. Table 2 and Fig. 13 depict the performance of the process alternatives in a low volume environment.

No longer is process $V I$ the first ranked technology, but $I V$ is the earnings leader in the low volume situation. This process earns about $\$ 8,000$ more than the second ranked process (laser beam cutting, III). That in turn is closely followed by process VI. A middle cluster is found with milling $(V)$ and process $I$. Process $I I$ has fallen behind with about half the value of the middle group and about a factor 2.5 in earnings behind the top group. This is surprising, initially, since modular tooling is often presented as a flexible production

Table 2 Results of the simulation for Scenario $A$ in the aerospace industry

\begin{tabular}{llll}
\hline Process & Earnings & Tool value & $\begin{array}{l}\text { Machine } \\
\text { utilization }\end{array}$ \\
\hline I & 113,597 & 597,289 & $0.2 \%$ \\
II & 61,688 & 473,112 & $0.2 \%$ \\
III & 154,698 & 9,937 & $1.9 \%$ \\
IV & 162,817 & 19,875 & $0.8 \%$ \\
V & 119,318 & 24,843 & $7.6 \%$ \\
VI & 154,635 & 149,059 & $0.3 \%$ \\
\hline
\end{tabular}

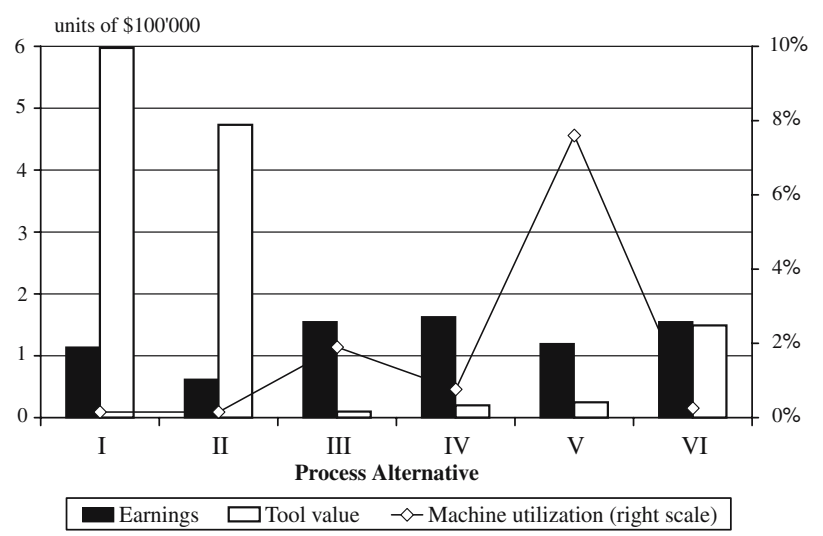

Fig. 13 Results of the simulation for Scenario $A$ in the aerospace industry 
strategy for low volume and high uncertainty, but in our case, the tool costs are too high to ever amortize this flexibility.

Two main drivers for this result can be determined. First, the tooling costs per part for the three stamping processes are about 10 times higher than the ones for laser beam cutting, punching, and milling. Second, the high switching times of processes $I$ and $I I$ cause, due to the tiny batch sizes, switching costs per part that are on the same order of magnitude as the parts sale prices and, thus, absorb the bulk of the products' margin.

Regarding the residual values of the tools, the ranking list looks the same as in the results discussed above. Process $I$ has the largest sunk tooling cost, followed by processes II and VI. Laser beam cutting has the best (lowest) value on its tool account, followed by punching and milling.

Generally, machine utilization is low, even the highest value is still below $10 \%$, this can be seen clearly in Fig. 13 , right hand scale.

As conclusion for firm $B$ the recommendation is to produce the levers by punching or laser beam cutting technology. Not only do these two process scenarios yield the most earnings, they also have an excellent 'earnings-to-tool value' ratio. Nevertheless, process VI would still be a viable process, especially if the firm has already an applicable press with enough free capacity. This is a remarkable result, since it indicates that the flexibility embedded in process VI (see Fig. 9) has given it a much greater volume range over which it can produce profitably (also recall volume flexibility defined by Sethi and Sethi, and Fig. 1).

\section{Sensitivity analysis}

Although the results differ clearly in most scenarios, the question is how robust the solutions are in terms of process ranking. So, a sensitivity analysis was formulated with the input parameters that are used in the simulation of market scenario A (see Fig. 11). Scenario $A$ was chosen because, first, it is the most likely market case. And second, its absolute results are most closely grouped, i.e., varying the input parameters in this scenario will likely have the biggest influence on the results and process rankings. The sensitivity analysis is carried out for all input variables for all six process alternatives in market scenario $A$. It is conducted 'ceteris paribus', which means that only a single parameter is changed at once while all other values remain fixed. Since we are mainly interested in a relative comparison of the processes, rather than absolute answers, this sensitivity analysis does not provide any information about the tendency and the amplitude of the result if an input parameter is perturbed by a small amount around the nominal solution. More usefully, it shows the range over which a simulation input parameter can be varied without changing the ranking of the results.
To determine the window of insensitivity of an input parameter, e.g., the output rate of process $I I$, the value of this variable is increased, and decreased, gradually, until the process ranking of both the earnings and the tool values change. In Fig. 14 the results of this analysis for the flexible process technology VI (flexible infeed) are shown.

Summarizing the results of the sensitivity analysis it can be said that process VI remains the winning technology in the high volume (automotive) case as long as:

- the costs for the tool in process VI do not exceed 179,833 ( $+20 \%$ from the current value),

- its output rate is not lower than 25 parts per minute $(-17 \%)$,

- the switching time for process $V I$ is not larger than $8.4 \mathrm{~min}$ $(+180 \%)$,

- the variable costs per hour do not exceed $\$ 69(+245 \%)$

- material utilization of process $V I$ is $96 \%$ or higher $(-4 \%)$,

- its scrap rate is not higher than $4 \%(+200 \%)$,

- the tool costs in process $I$ are not below $\$ 48,371(-68 \%)$ for tool $a, \$ 114,406$ for tool $b(-38 \%)$, and $\$ 63,394$ $(-75 \%)$ for tool $c$,

- the output rate of process $I$ does not exceed 70 parts per minute $(+40 \%)$, or

- process I's process' switching time is not faster than 9 min $(-40 \%)$.

The listing above is an 'OR'-statement and not an 'AND'conclusion. Nothing is said about the robustness of the solution while changing more than one parameter at a time. Nevertheless, the winning solution, process $V I$, appears to be relatively stable. It appears that embedding flexibility in process parameters, combined with a clever tooling concept, can outperform a more expensive modular tooling strategy (process II) in this case.

\section{Conclusions}

This paper presented a simulation-based framework for evaluation and ranking of various flexible and non-flexible component manufacturing processes. A case study using a family of simple metallic parts was worked out to show how different market and product aspects influence the decisions for defining the optimal manufacturing processes. Uncertainty is modeled in three market scenarios, each with different specification and demand fluctuations. These market scenarios are combined with six different process alternatives, each modeling a different manufacturing process. This is done for both, a hypothetical automotive (high volume) and aerospace (low volume) environment. 
Fig. 14 Range of input parameters for market scenario $A$ and process $V I$ for invariant ranking

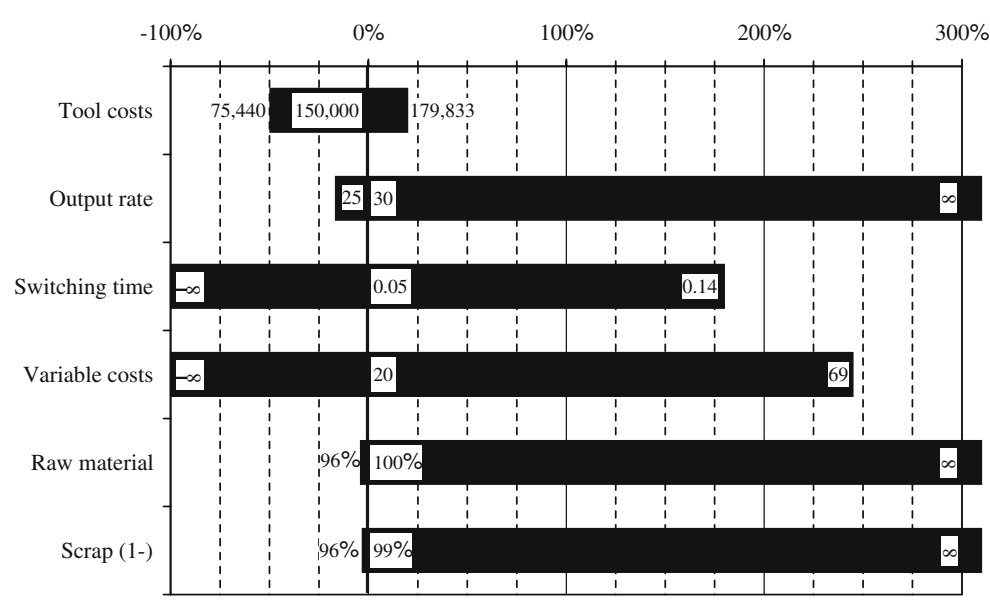

The results also show that maximizing parts manufacturing flexibility is not the most profitable strategy. In Fig. 16 the earnings of the different process scenarios are compared relative to each other.

While in the low volume scenario all analyzed processes generate similar earnings, in the high volume case, only fast processes can run profitably. Even though process scenarios $I I I, I V$, and $V$ possess higher process flexibility than the other three, it is obvious that this flexibility is no longer profitable at higher volume levels.

Moreover, and this is a central point, if the variety of part's characteristics can be reduced to one or two dimensions, such as the length in the lever example (Fig. 4), then flexibility can be easier to embed in processes which are also capable of high volumes. The key question, then, is how to determine those one or two dimensions which should be designed to be flexible.

The 5-step simulation-based method (Fig. 3) presented in this paper is primarily targeted for use in strategic decision making in an industrial manufacturing context. The simulation results and metrics primarily help to make three types of decisions:

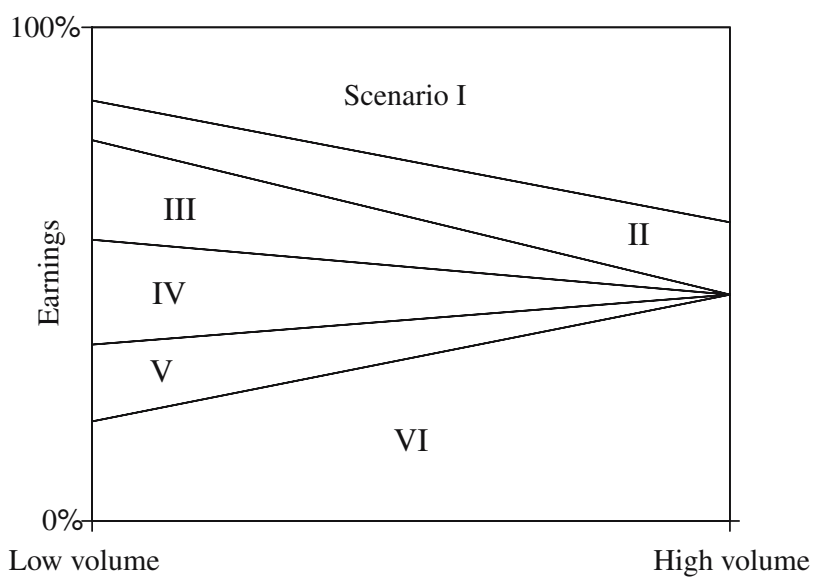

Fig. 16 Relative earnings of processes versus number of units produced 
1. Given component specifications and uncertain demand estimates, help select from among a set of potential manufacturing processes the ones that will maximize earnings, minimize sunk investment and minimize opportunity costs.

2. Given long range estimates and strategic goals, help prioritize investments in new component manufacturing equipment and focus efforts to increase manufacturing flexibility.

3. Given a set of fabrication equipment and market scenarios, optimize the product mix and number of variants to best take advantage of existing manufacturing capabilities.

\section{Future research}

The work described in this article points towards further research concerning parts manufacturing flexibility. The direction pointed out by the simulation is an integrated optimization and evaluation framework for flexible component manufacturing systems. In such an integrated approach, the exogenous uncertainties of future production volumes and parts specifications have to be projected onto an entire manufacturing plant, not just on a single machine thread as was done in Section 'Case study'.

Then, a more accurate framework for the measurement of flexibility can be built. The simulation methodology compiled in this paper can serve a broader decision support system for valuing flexibility which will help compare different flexibility strategies. If additional market scenarios, processes and multi-year time periods must be considered one will not be able to resort to a full combinatorial evaluation as was done here, but will enlist various dynamic, multi-objective and stochastic optimization techniques.

Based on process alternative $V I$, further research on flexibility in the process parameters and raw materials should be done. It appears that embedding flexibility in materials and process parameters outperforms the flexible modular tooling approach, but it is unclear whether this conclusion holds more generally, because it depends strongly on the particular processes and ratios of variable costs to capital investments in tooling as well as switching time and cost. Nevertheless, a methodology for determining the crucial dimensions of a part, in regards to making those attributes flexible, will be very useful in order to design more intelligent processes in the future.
Mills' quadratic cost function (Eq. 1) deserves some more research, considering the analysis of parameters $\alpha, \beta$, and $\delta$ as well as their calibration using industrial data. A table which provides these constants for a large set of current manufacturing processes would help production managers and engineers select the best manufacturing processes to use for their components.

\section{References}

Fricke, E., Schulz, A. P., Wenzel, S., \& Negele, H. (2000). Design for changeability of integrated systems within a hyper-competitive environment, Colorado 2000 Conference "Systems Approach to Product Innovation and Development in Hyper-Competitive Environments", INCOSE

Gupta, Y. P., \& Somers, T. M. (1992). The measurement of manufacturing flexibility. European Journal of Operational Research, 60, 166-182.

Hauser, D. P. S. (2004). Flexibility in aerospace and automotive Component manufacturing Systems: Practice, strategy and optimization, Diplomarbeit, S.M. thesis. Institute for Virtual Production, Swiss Federal Institute of Technology, ETH Zurich, Switzerland.

Mills, D. E. (1984). Demand fluctuations and endogenous firm flexibility. The Journal of Industrial Economics, 33(1), 55-71.

Mehrabi, M. G., Ulsoy, A. G., \& Koren Y. (2000). Reconfigurable manufacturing systems: Key to future manufacturing. Journal of Intelligent Manufacturing, 11(4), 403-419.

Pine II, B. J. (1993). Mass customization: The new frontier in business competition. Boston: Harvard Business School Press.

Schips, B. (2000). Einführung in die Volkswirtschaftslehre, Vorlesungsunterlagen. ETH Zürich: Institut für Wirtschaftsforschung.

Schulz, A. P., \& Fricke E. (1999). Incorporating flexibility, agility, robustness, and adaptability within the design of integrated systems-key to success? 18th DASC, Gateway to the New Millenium. IEEE.

Sethi, A. K., \& Sethi, S. P. (1990). Flexibility in manufacturing: A survey. The International Journal of Flexible Manufacturing Systems, 2, 289-328.

Simpson, T. W., Siddique, Z., \& Jiao, (Roger) J. (2006). Product platform and product family design: Methods and applications, New York: Springer Verlag.

Suh, E. S., Kim, I. Y., \& de Weck, O. L., (2004). Design for Flexibility: Performance and Economic Optimization of Product Platform Components, AIAA-2004-4310, 10th AIAA/ISSMO Multidisciplinary Analysis and Optimization Conference, Albany, New York.

Upton, D. M. (1994). The management of manufacturing flexibility. California Management Review, 36(2), 72-89. 